\title{
Identification of Jun loss promotes resistance to histone deacetylase inhibitor entinostat through Myc signaling in luminal breast cancer
}

\author{
Maki Tanioka ${ }^{1,2}$, Kevin R. Mott ${ }^{1}$, Daniel P. Hollern' ${ }^{1}$, Cheng Fan ${ }^{1}$, David B. Darr ${ }^{1,3}$ and Charles M. Perou ${ }^{1,2^{*}}$
}

\begin{abstract}
Background: Based on promising phase II data, the histone deacetylase inhibitor entinostat is in phase III trials for patients with metastatic estrogen receptor-positive breast cancer. Predictors of sensitivity and resistance, however, remain unknown.

Methods: A total of eight cell lines and nine mouse models of breast cancer were treated with entinostat. Luminal cell lines were treated with or without entinostat at their $I_{50}$ doses, and MMTV/Neu luminal mouse tumors were untreated or treated with entinostat until progression. We investigated these models using their gene expression profiling by microarray and copy number by arrayCGH. We also utilized the network-based DawnRank algorithm that integrates DNA and RNA data to identify driver genes of resistance. The impact of candidate drivers was investigated in The Cancer Genome Atlas and METABRIC breast cancer datasets.
\end{abstract}

Results: Luminal models displayed enhanced sensitivity to entinostat as compared to basal-like or claudin-low models. Both in vitro and in vivo luminal models showed significant downregulation of Myc gene signatures following entinostat treatment. Myc gene signatures became upregulated on tumor progression in vivo and overexpression of Myc conferred resistance to entinostat in vitro. Further examination of resistance mechanisms in MMTV/Neu tumors identified a portion of mouse chromosome 4 that had DNA copy number loss and low gene expression. Within this region, Jun was computationally identified to be a driver gene of resistance. Jun knockdown in cell lines resulted in upregulation of Myc signatures and made these lines more resistant to entinostat. Jun-deleted samples, found in 17-23\% of luminal patients, had significantly higher Myc signature scores that predicted worse survival.

Conclusions: Entinostat inhibited luminal breast cancer through Myc signaling, which was upregulated by Jun DNA loss to promote resistance to entinostat in our models. Jun DNA copy number loss, and/or high MYC signatures, might represent biomarkers for entinostat responsiveness in luminal breast cancer.

\section{Background}

Despite advances in early detection and perioperative treatments, breast cancer remains the second leading cause of cancer deaths among women in developed countries [1]. This is because a certain portion of the

\footnotetext{
* Correspondence: cperou@med.unc.edu

'Lineberger Comprehensive Cancer Center, University of North Carolina at Chapel Hill, Chapel Hill, NC 27599, USA

2Department of Genetics, University of North Carolina, Chapel Hill, NC 27599, USA

Full list of author information is available at the end of the article
}

patients continue to develop fatal metastatic disease and eventually succumb to death. Estrogen receptor (ER)-positive tumors comprise $70 \%$ of the breast cancer population and most of them respond to aromatase inhibitors (AIs); however, others acquire resistance. Currently, the combinations of CDK4/6 inhibitors, or a mTOR inhibitor with AIs, have shown improvement of progression-free survival in patients with metastatic ER-positive breast cancer [2-4]. Unlike these agents, entinostat, a class I histone deacetylase (HDAC) inhibitor, may augment response to endocrine therapy. HDAC inhibitors have

(c) The Author(s). 2018 Open Access This article is distributed under the terms of the Creative Commons Attribution 4.0 International License (http://creativecommons.org/licenses/by/4.0/), which permits unrestricted use, distribution, and 
been thought to induce histone acetylation leading to transcriptional re-activation of epigenetically inactivated cancer-associated genes which suppress cell proliferation and promote apoptosis [5]. Class I HDAC isozymes include $\mathrm{HDAC} 1-3$, whose expression has been shown to be increased in hormone receptor-positive or high-grade tumors [6]. Indicating the importance of epigenetic modification in the context of endocrine therapy, entinostat with letrozole combination therapy was able to reduce the volume of letrozole-resistant tumors [7]. From a safety standpoint, entinostat does not target class II HDACs which are expressed in the heart [8]; therefore, this selectivity of entinostat may eliminate serious side effects such as QT-prolongation and cardiac infarction that have been associated with pan-HDAC inhibitors $[9,10]$. For postmenopausal women with metastatic ER-positive breast cancer, a randomized phase II trial of entinostat showed benefits in both progression-free and overall survival and the incidence of reported cardiac disorders was similar between entinostat and placebo arms [11]. With these promising results, entinostat received breakthrough designation from the Food and Drug Administration (FDA), and currently, a phase III registration trial E2112 (NCT02115282) is ongoing.

Some HDAC inhibitors, Vorinostat, Panobinostat, Belinostat, and Romidepsin, have been granted FDA approval for cancer, yet there are no validated markers for their clinical decision making. Although c-Myc has been shown to be a key target for sensitivity to other HDAC inhibitors in various cancers [12-15], it is still unclear whether absolute transcriptional or genomic levels of c-Myc (hereafter, Myc) predict sensitivity to all HDAC inhibitors. In addition, Myc remains difficult to target with small molecule Myc inhibitors despite numerous attempts. As for entinostat in breast cancer, a number of molecular features have been suggested as underlying mechanisms of response. This includes upregulation of ER [7], downregulation of Akt [16], upregulation of E-cadherin [17], and functional inhibition of myeloid-derived suppressor cells $[18,19]$. However, studies detailing tumor responses to entinostat are still needed to identify modes of sensitivity and resistance. Here we studied entinostat's mechanism of action using multiple models of luminal and non-luminal breast cancers and identified two possible biomarkers of resistance.

\section{Methods}

\section{Breast cancer cell lines}

The human breast cancer cell lines were maintained in standard growth media (SKBR3, BT474, MCF-7, T47D) in RPMI (Gibco) plus 10\% FBS (Sigma) and anti-biotic anti-mycotic (Gibco) or in DMEM (Gibco, high glucose) with 10\% FBS (MDA231) [20]. Hs578t and WHIM12 cell lines were cultured in HuMEC media with supplements
(Gibco) plus Bovine Pituitary Extract (Gibco) and antibiotic anti-mycotic (Gibco). WHIM12 is a patient-derived xenograft cell line obtained from Matthew Ellis (Baylor College of Medicine). All cell lines were grown at $37^{\circ} \mathrm{C}$ and $5 \%$ carbon dioxide and were obtained from the American Type Culture Collection unless otherwise specified.

\section{Cell proliferation assay}

The effects of entinostat on the proliferation of various human or mouse cancer cell lines were determined by using the [3-(4,5-dimethylthiazol-2-yl)-5(3-carboxymethoxyphenyl)-2-(4-sulfophenyl)2H-tetrazolium [21] assay (CellTiter $96^{\circ}$ AQueous One Solution Cell Proliferation Assay, Promega), except for T47D where the effects were measured using Cell titer glo (Promega). A total of 3000-5000 cells were seeded into 96-well culture plates and treated with entinostat (Sigma) for $72 \mathrm{~h}$ and then treated with MTS for $2 \mathrm{~h}$. Cell viability was determined by measuring the absorbance at $490 \mathrm{~nm}$. Six replicates for each time point were measured. The generation of dose-response curves was performed using the GraphPad Prism.

\section{Genetically engineered mouse models (GEMMs)}

GEMMs of strain FVB carrying a transgene for rat HER2 (MMTV/Neu) [22] and C3 SV40 T-antigen (C3tag) [23], or patient-derived xenografts (PDXs) WHIM8 and WHIM35 [24] in Nod-Skid-Gamma (NSG) mice were bred in-house and observed until the onset of a mammary tumor approximately $0.6 \mathrm{~cm}$ in any dimension. Tumors derived from TP53 -/- mammary gland transplant lines (T2, T11, 2225L, 2208L, and 2396R) [25] were passaged in BALB/c wild-type mice by subcutaneous injection of one half million cells resuspended in Matrigel into the mammary fat pad as previously described [26]. A minimum tumor volume of approximately $0.6 \mathrm{~cm}$ in size was used as the starting point for treatment studies; $12-28$ mice for each experiment were randomized into untreated or treated groups and monitored with tumor growth measurements (Additional file 1: Figure S1). For C3tag and MMTV/Neu mice, we added information on 30 or more mice using historical controls, as well as contemporaneous controls. Tumor volumes were measured by caliper as (width) $\times($ length $)^{2} / 2$ for MMTV/Neu or C3tag and $\pi / 6 \times$ (length) $^{3}$ for transplant lines. Entinostat (Sigma) was milled into chow at $12 \mathrm{mg} / \mathrm{kg}$, which was determined by in-house dose-finding study of entinostat. For short-term response, tumor size was measured at baseline for all the models and at 7-day point for 2225L, 10-day point for 2396R and T2, 14-day point for 2208L, and 21-day point for WHIM8, WHIM35, T11, C3tag, and MMTV/Neu. Treatment periods were dependent on inherent tumor line growth rates, and the percent change in volume was used to quantify response. For 
survival, treatment with entinostat was started at time zero (i.e., tumor $0.6 \mathrm{~cm}$ ) and continued until either the mouse developed a tumor burden sufficient to warrant euthanasia ( $2 \mathrm{~cm}$ in any dimension) or until weight loss totaling $20 \%$ of the initial starting body mass was observed. All work was done under protocols approved by the University of North Carolina (UNC, Chapel Hill, NC) Institutional Animal Care and Use Committee.

\section{DNA, RNA, and protein extraction}

Total RNA was purified using the RNeasy Mini kit (Qiagen), and DNA was extracted using the DNeasy Kit (Qiagen). We extracted nuclear protein using NE-PER nuclear and cytoplasmic extraction reagents (Thermo Scientific). Following extraction, protein concentration was determined using Micro BCA protein assay kit (Thermo Scientific).

\section{Gene expression and signature analysis}

A total of $1 \mu \mathrm{g}$ human RNA or $2 \mu \mathrm{g}$ of mouse RNA were purified and profiled as described previously using DNA oligo microarrays (Agilent Technologies, USA) [27]. The LOWESS normalized log2 ratios (Cy5 sample/Cy3 control) of probes mapping to the same gene (Entrez ID) were averaged to generate gene-level expression estimates. Gene expression changes were measured in luminal cell lines SKBR3 (ER-/HER2+), BT474 (ER+/HER2+), and MCF7 (ER+/HER2-) treated with or without entinostat at their $\mathrm{IC}_{50}$ doses. Significantly differentially expressed genes were identified (false discovery rate of 0\%) using unpaired two-class significance analysis of microarrays (SAM) [28] to compare untreated vs. entinostat-treated samples. Hierarchical clustering using 814 of The Genome Cancer Atlas [29] breast cancer samples was done using Gene Cluster 3.0 [30]. For hierarchical clustering analyses, the genes/rows were median centered, and clustering of arrays was conducted with correlation centered genes and arrays, and centroid linkage. Array cluster viewing and display were conducted using JavaTreeview v1.1. 5r2 [31]. We defined "signatures" as any gene set that contained a minimum of 10 genes and a Pearson node correlation greater than 0.5 in this TCGA breast cancer hierarchical cluster dataset.

We investigated the significance of gene signatures using the "Investigate Gene Sets" method of Gene Set Enrichment Analysis (GSEA, http://software.broadinstitute. org/gsea/msigdb/annotate.jsp) [32]. We also applied a collection of 517 publicly available, gene expression signatures, representing multiple biological pathways and cell types as well as entinostat signatures [33]. These 517 signatures have all been published [34] and obtained from 73 publications or GSEA [32] and partially summarized by Fan et al. [35]. Using the TCGA data, we applied each signature to the dataset in a manner consistent with their derivation.
For 480 signatures with homogeneous expression across genes, we used the median expression value from all genes within a signature, and 37 signatures were based on correlation to predetermined gene centroids or based on pre-specified published algorithms.

Using the gene expression data from SKBR3, BT474, and MCF-7 treated with or without entinostat, we also investigated three $M y c$ gene signatures which represents Myc signaling: MYC.1PFDR_UP [21] and MYC.2012 [36] derived from breast cancer mouse models comparing MMTV-Myc and other models, and DUKE.MODULE13 [37] comparing Myc-overexpressed versus control human mammary epithelial cells. Genes in mouse and human version of signatures are shown in Additional file 2: Table S1. We used the median expression value from all genes in each signature as a gene signature score.

In addition, a total of 27 MMTV-Neu mouse tumors (luminal subtype) were untreated $(N=8)$ or treated with entinostat at $12 \mathrm{mg} / \mathrm{kg}$ for 3 weeks $(N=5), 6$ weeks $(N=6)$, or until progression after complete response $(N=8)$. We profiled gene expression of these tumors and calculated $M Y C$ gene signature scores for each subgroup. Using the Mouse Genome Database (http://www.informatics.jax.org/ homology.shtml), the lists of human $M Y C$ gene signatures were converted to orthologous mouse genes.

\section{Lentiviral transfection}

To determine whether Myc or Jun levels influence the effects of entinostat on cell viability, we transfected luminal breast cancer cells with lentiviruses expressing the non-degradable, phosphorylation mutant (T58A) of $M y c$ or two kinds of shRNA targeting Jun. To overexpress MycT58A or mCherry, constructs in pLEX-MCS-puro vector (Thermo Scientific) were kindly provided by Gary L Johnson [9]. HEK293T cells were transfected with Lipofectamine 2000 (Invitrogen) with pLEX-MCS-puro constructs and packaging plasmids. Forty-eight hours after transfection, viral supernatant was collected and filtered through $0.45-\mu \mathrm{m}$ syringe filters. To knockdown Jun expression, shRNA for Jun and scramble DNA as a control were obtained from Cyagen and the pLV-Puro-U6 vector was used. Target sequences for Jun knockdown were ATTCGATCTCATTCAGTATTA or TTCTGGCCT GCCTTCGTTAAC at 3'UTR of Jun (i.e., two different RNAi targeting constructs). After SKBR-3 or MCF-7 cells were transduced with lentivirus-mediated mutant $M y c$ with in the presence of $6 \mu \mathrm{g} / \mathrm{ml}$ polybrene, the cells were incubated in various doses of entinostat for $72 \mathrm{~h}$, and then the viability of cells was measured using the MTS assay. Likewise, BT474 or T47D cells were transfected with lentivirus-mediated shRNA for Jun.

Expression levels of Myc or Jun protein in nucleus were determined by Western blot following recommendations of antibody suppliers. Antibodies used were 
HA-tag for exogenous Myc (C29F4, Cell Signaling), endogenous Myc (D3N8F, Cell Signaling), Jun (60A8, Cell Signaling), and beta-actin (\#4967, Cell Signaling) as a housekeeping gene. The relative chemiluminescent intensities were quantified in individual frames using ImageJ software $(\mathrm{NIH})$.

\section{Assessment of DNA copy number changes in MMTV/Neu tumors}

To investigate DNA copy number changes, we used the custom HD-array Comparative Genomic Hybridization $(\mathrm{aCGH})$ platform which was designed and built on the Mouse 244 k Custom Oligo platform (GPL15359 Agilent UNC Perou Lab $1 \times 244 \mathrm{k}$ Custom Tiling CGH Array). Two hundred thirty thousand six hundred and six probes cover a total region of $45 \mathrm{Mb}$, and this design gives an average resolution of $200 \mathrm{bp}$ between contiguous probes. Labeling and hybridization were performed according to the manufacturer's instructions using the Agilent Genomic DNA Labeling Kit PLUS (Catalog Number 5188-5309). One microgram of DNA from liver or spleen of FVB strain mouse was used as normal reference DNA, which was compared versus $1 \mu \mathrm{g}$ of DNA from every mouse tumor sample. Microarrays were scanned on an Agilent DNA Microarray scanner (G2565CA) and the data uploaded to the University of North Carolina Microarray Database (www.genome.unc.edu). To determine regions of Copy Number Aberration (CNA), we utilized the R package SWITCHdna [38], which can identify breakpoints in aCGH data. SWITCHdna detects transition points that maximize the $F$ statistic and have regions on either side of the breakpoint that are larger than $250 \mathrm{~kb}$. Following detection of the transition points, a $\log 2$-ratio segment average value and corresponding $z$-score are determined, along with the number of observations used. In this study, we used a $\mathrm{z}$-score of 3 and a minimum intensity measurement of 0.09 . The end results are the identification of segments of CNA, along with a quantitative value for that copy number change (i.e., loss or gain). All subsequent plots were produced after applying this significance filter to our data. These segment-level copy number values were changed into gene level using R package Switchplus [39].

\section{DawnRank analysis}

We used the DawnRank algorithm [40] as a novel computational method that uses within-tumor integrated analyses of DNA aberrations in context of RNA expression that is used to population predetermined protein-protein networks in order to find possible individual driver genes that might predict resistance to entinostat. Using the DawnRank predefined protein-protein interaction networks, we populated this network with mRNA gene expression data for each sample and calculated a score for each gene based upon expression of the genes connected to it in the network. Using somatically altered genes with CNA data described above, we applied DawnRank to four groups of the MMTV-Neu mouse tumors which were untreated $(N=6)$, or treated with entinostat at $12 \mathrm{mg} / \mathrm{kg}$ for 3 weeks $(N=4), 6$ weeks $(N=4)$, or until progression after complete response $(N=8)$ according to the "percentrank" analysis mode, which aggregates the DawnRank results across a predefined set of samples.

\section{TCGA and METABRIC samples}

Two independent publicly available human breast cancer datasets, TCGA [29] and METABRIC [41], were investigated to validate the impact of Jun deletion. We used PAM50 intrinsic subtypes, mRNA expression, and DNA copy number changes in 814 primary breast tumors from TCGA. PAM50 intrinsic subtypes and mRNA expression data are publicly available at cBioPortal website (http://cancergenome.nih.gov/). Copy number variation across the genome was determined as follows: The sequence reads were aligned to the genome (hg 19) using the bwa mem algorithm (https:/github.com/lh3/bwa; v0.7.4) with the default parameters. Duplicates were removed using Picard (http://broadinstitute.github.io/picard/). Quality statistics were also generated with Picard including measures of fragment length, sequence content, alignment, capture bias and efficiency, coverage, and variant call metrics. Copy number assessments were performed using SynthEx [42]. In brief, counts data for fixed $100-\mathrm{kb}$ bin were generated using BEDTools [43]. The read ratios were calculated using the "synthetic normal" strategy described in SynthEx. A trending filter procedure was applied to segment the genome. The segment-level copy number values, which is the $\log 2$ ratios of normalized signal intensities between tumor and reference, were finally corrected by purity and ploidy estimates from SynthEx, taking whole genome doubling into account for these values. These segment-level values were changed into gene-level using Switchplus [39]. Copy number values derived from exome sequencing were compared with those from SNP6.0 among the TCGA samples [44] with ploidy $1.75-2.5$, then the thresholds for gain or loss were determined as 0.25 or -0.32 , respectively [42]; we applied these thresholds to copy number values on the TCGA samples to call gained and lost segments. All genomic data, including DNA copy number and gene expression, have been deposited into the GEO (series ID GSE118744).

The METABRIC human breast cancer dataset includes breast cancer-specific survival data as well as gene expression and DNA copy number data of 1992 resected primary breast tumors. All clinical and genomic data are also publicly available at the cBioPortal. Copy number datasets within the portal are generated by GISTIC [45] to determine the copy number status of each gene in each sample. Amplification or deletion was determined 
by applying both low- $(-1,-2)$ and high-level $(+1,+2)$ thresholds to the gene copy levels of all the samples. In our study, Jun copy number loss was defined by " -2 " (possibly a homozygous deletion) and "- 1 " (possibly a heterozygous deletion). To investigate the clinical impact of three Myc signatures (Myc_1pFDR, Myc.2012, or Duke_Module13_myc), the patients with luminal A or luminal B PAM50 subtype [46] breast cancers were classified into three rank order groups according to scores from each Myc signature. To investigate clinical impact of Jun copy number loss, the patients were classified into groups with/without Jun copy number loss, and breast cancer-specific survival was assessed by Kaplan-Meier curves. Patients with survival of $>20$ years were excluded. Two-sided log-rank tests and univariate Cox regression analyses were conducted to determine significance of each endpoint.

Statistical analyses on signature scores, box plots, SAM, DawnRank, and Kaplan-Meier curves of human samples were performed using $\mathrm{R}$ version 3.1.2, while generation of survival curves or response plots on mouse models, and $\mathrm{IC}_{50}$ curves on cell lines were performed using the GraphPad Prism version 7 software (GraphPad Software, San Diego).

\section{Results}

Sensitivity to entinostat among breast cancer models in vivo and in vitro

Given the promising phase II results for entinostat, our study sought to clarify the mechanism(s) of entinostat sensitivity and to find biomarker(s) to predict its sensitivity or resistance in human breast cancer. As a first step, we investigated preclinical models of breast cancer for entinostat sensitivity. To examine sensitivity in vitro, we identified the $\mathrm{IC}_{50}$ doses of entinostat for seven human breast cancer cell lines (Fig. 1a). Cell lines with luminal features (BT474, MCF-7, SKBR3, and T47D) were more sensitive to entinostat compared with basal-like or claudin-low (MDA231, Hs578t, and WHIM12) cell lines. Luminal model sensitivity was confirmed in vivo by testing murine luminal, basal-like, claudin-low TP53-/GEMMs, and PDX models, with entinostat at $12 \mathrm{mg} / \mathrm{kg}$ (Fig. 1b). Entinostat generally inhibited tumor growth for most murine models, irrespective of subtype, but only the luminal MMTV/Neu murine tumors presented a complete reduction in tumor volume after treatment of entinostat. In addition, we assessed the effects of entinostat on the overall survival of tumor-bearing mice. Although entinostat generally prolonged the survival for mice with any of the tumor types, entinostat greatly extended lifespan from a median of 29 to 123 days in MMTV/Neu (Additional file 1: Figure S1). Figure 1d shows a time course of eight MMTV/Neu tumors from six mice; although they initially responded to entinostat completely, recurrent tumors emerged after the 5-10 weeks of entinostat treatment. In addition, we note some tumors (180226-Lt.Neck and 180,165-Lt.inguinal tumors) appeared after a long latency, perhaps suggesting initial repression of tumorigenic cells that emerged with acquired resistance.

\section{Global gene expression change caused by entinostat in luminal breast cancer}

To identify signatures of entinostat-regulated genes, gene expression analyses were performed. Luminal cell lines SKBR3 (ER-/HER2+), BT474 (ER+/HER2+), and MCF7 (ER+/HER2-) were treated with or without entinostat at their $\mathrm{IC}_{50}$ doses for $72 \mathrm{~h}$ and their gene expression profiles determined. Differential expression analysis between untreated vs entinostat-treated cell lines revealed 563 upregulated and 565 downregulated genes with FDR of $0 \%$ (Additional file 2: Table S2-A). We hierarchically clustered these two gene lists using 817 TCGA breast cancer samples, then defined "signatures" as any gene set that contained a minimum of 10 genes and a Pearson node correlation of greater than 0.5. Using these criteria, we defined six upregulated signatures and three downregulated signatures (Fig. 2a, all genes in signatures listed on Additional file 2: Table S2-B). We next investigated the potential impact of each signature on the survival among luminal breast cancer samples in METABRIC (Additional file 1: Figure S2-A). In addition, we plotted the scores for each entinostat signature according to PAM50 intrinsic subtypes using TCGA breast tumors (Additional file 1: Figure S2-B). Figure $2 \mathrm{~b}$ shows the summary of the characteristics of all signatures. Within the downregulated gene signatures, down signature 1 (285 genes) had significant correlations with HALLMARK_MYC_TARGETS_v1 (q-value, $2.7 \mathrm{e}^{-17}$ ) and HALLMARK_MYC_TARGETS_v2 (q-value, $\left.1.7 \mathrm{e}^{-4}\right)$ in the GSEA "Investigate gene set" analysis. Similarly, when TCGA samples were scored for these entinostat signatures with 517 other signatures, clustering analysis revealed the down signature 1 was correlated with three Myc signatures (MYC.1PFDR_UP, MYC.2012, and DUKE.MODULE13) as well as many proliferation signatures (Pearson correlation, 0.64).

\section{Entinostat inhibits luminal breast cancer through Myc signaling}

Figure 3a illustrates that Myc signature scores goes down after treatment with entinostat at their $\mathrm{IC}_{50}$ doses in three luminal human cell lines. Myc is well appreciated as an oncogene that has been shown to induce HDAC2 (a major target of entinostat) in colorectal [47] and pancreatic cancer [12]; therefore, we sought to experimentally validate the role of Myc signaling in sensitivity to entinostat. Using lentiviral transfection of Myc 

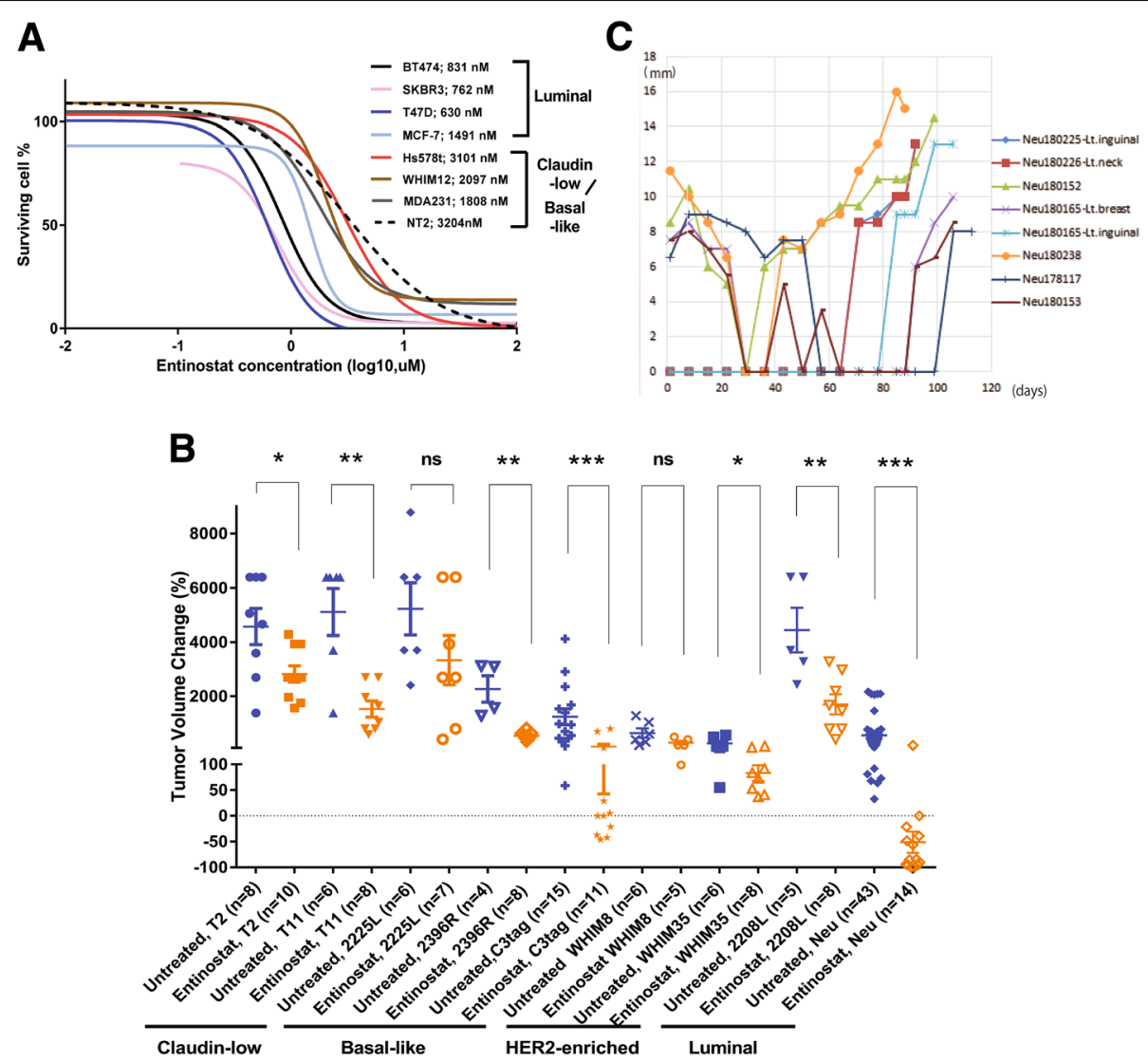

Fig. 1 Luminal breast cancer is sensitive to entinostat in vivo and in vitro. a Antiproliferative activities of entinostat in breast cancer cells. The effects of entinostat on the proliferation of various human or mouse cancer cell lines were determined by using the [3-(4,5-dimethylthiazol-2-yl)5-(3-carboxymethoxyphenyl)-2-(4-sulfophenyl)2H-tetrazolium [21] assay (CellTiter 96 ${ }^{\circledR}$ AQueous One Solution Cell Proliferation Assay, Promega). Cells were seeded into 96-well culture plates and treated with entinostat for 3 days, and then treated with MTS for $2 \mathrm{~h}$. Cell viability was determined by measuring the absorbance at $490 \mathrm{~nm}$. Inhibitory concentration (IC) curves are shown with $\mathrm{IC}_{50}$ values in legend. b Short-term treatment responses for seven mouse models of mammary cancer. The models used were p53 null T2 and T11 which were chosen based on their similarity in gene expression to claudin-low subtype, 2396R, 2225L, and C3tag whose subtype was basal-like, WHIM8 and WHIM35 whose subtypes were HER2-enriched [24], and 2208L and MMTV/Neu whose subtypes were luminal [25]. Entinostat at $12 \mathrm{mg} / \mathrm{kg}$ was continuously administered via the chow. Tumor size was measured at baseline for all the models and at 7-day point for 2225L, 10-day point for 2396R and T2, 14-day point for 2208L, and 21-day point for T11, WHIM8, WHIM35, C3tag, and MMTV/Neu. Treatment periods were dependent on their faster growth rate. The change in tumor volume is plotted as whisker plots shown as measures of tumor responsiveness for entinostat-treated models with their matched untreated control. The error bars represent the $95 \% \mathrm{Cls}$. The number of animals in each treatment group is indicated in parentheses. Mann-Whitney tests were conducted between untreated control and entinostat-treated group for each mouse model. ${ }^{*},<0.05 ;{ }^{* *},<0.01 ;{ }^{* *},<0.001$; ns, not significant. c A total of eight MMTV/Neu tumors from six mice showed complete response but finally became resistant to entinostat chow (12 mg/kg). 180226-Lt.Neck or 180165-Lt.inguinal were not detected at the beginning but appeared after 63 or 77 days of treatment, respectively

or a phosphorylation incompetent mutant $\mathrm{Myc}^{\mathrm{T} 58 \mathrm{~A}}$, we tested the impact of Myc overexpression on entinostat sensitivity. Western blot analysis confirmed Myc overexpression, and quantification revealed that protein levels of Myc were 3.2- and 16.5-fold higher than control cells in SKBR3 and ZR75-1, respectively. Similarly, $\mathrm{Myc}^{\mathrm{T} 58 \mathrm{~A}}$ overexpressed cells had 5.4-, 1.4-, and 5.4-fold higher Myc protein levels than control cells in SKBR3, MCF7, and ZR75-1, respectively (Additional file 1: Figure S3-A \& S3-C). Importantly, we observed that Myc and $\mathrm{Myc}^{\mathrm{T} 58 \mathrm{~A}}$ overexpression in SKBR3, MCF-7, and ZR75-1 cells increased resistance to entinostat (Fig. 3b-d).
Examining gene expression profiles of MMTV/Neu tumors responding to entinostat at a 3-week time point confirmed a reduction in expression of $M y c$ and Myc target genes. Myc signature scores stayed in a low level at the 6 -week point but increased when the tumors became resistant to entinostat (Fig. 3e, the changes in tumor size are shown in Additional file 2: Table S3). Further, $M y c$ gene signatures did not change between 16 untreated vs 5 treated C3tag tumors, which did not respond to entinostat for 3 weeks $(p=0.99$, data not shown). Taken together, these results demonstrate Myc as a possible target of entinostat therapy and point to 


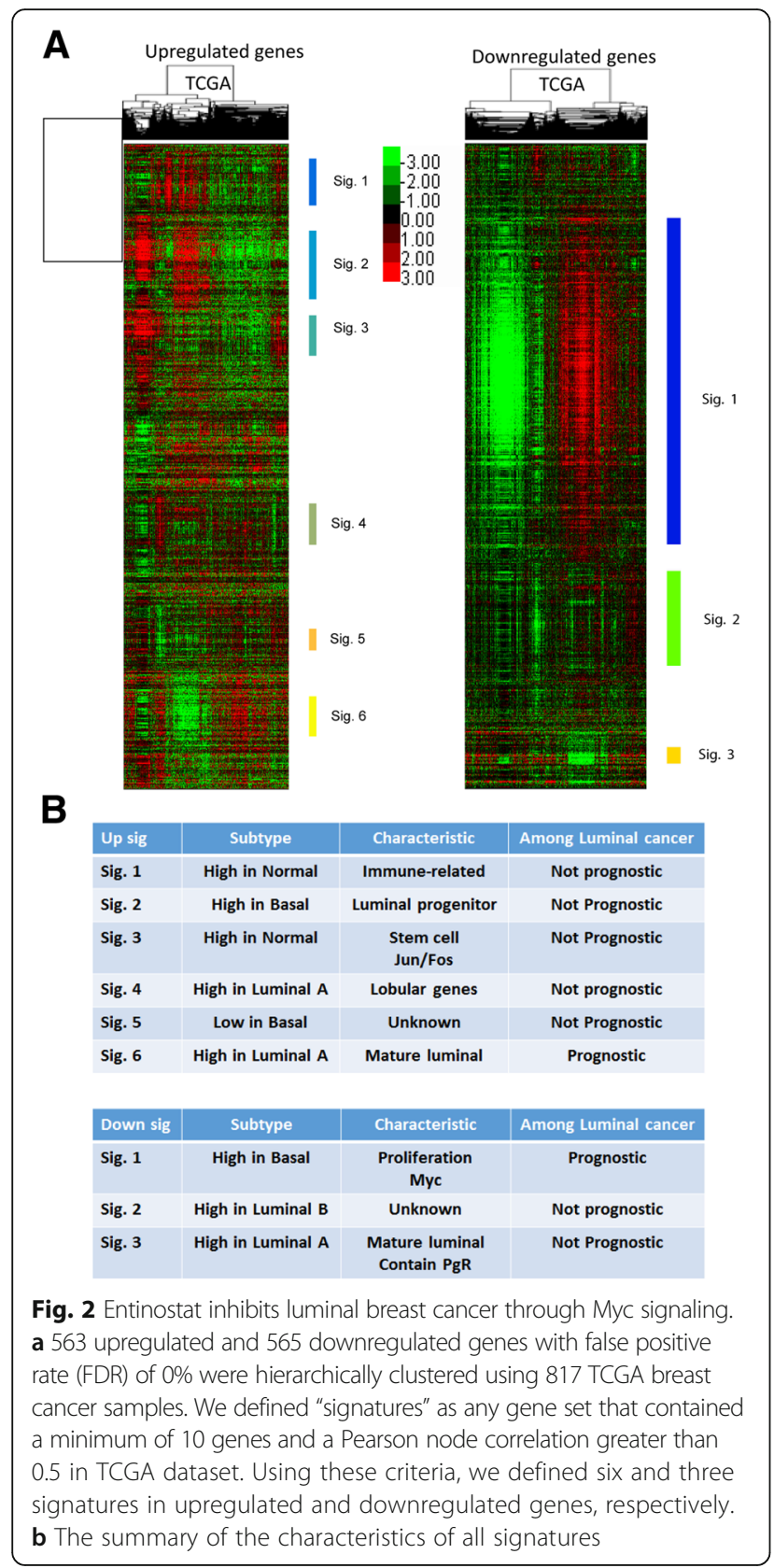

repression of Myc target genes as a critical mediator of sensitivity.

\section{Genomic Jun loss causes resistance to entinostat}

To identify potential mechanisms of resistance, we analyzed MMTV/Neu sensitive and resistant tumors by aCGH (Fig. 4a). Copy number aberration in untreated tumors was few. However, a large portion of mouse chromosome 4 showed DNA copy number loss in tumors that progressed while on entinostat for a long time. This observation led us to hypothesize that copy number loss at Chr.4 conferred resistance to entinostat. To test for driver genes responsible for the resistance, we used
DawnRank analysis [40] to integrate copy number data with the gene expression data from MMTV/Neu tumors that were untreated, treated for 3 weeks, treated for 6 weeks, or treated until progression. Table 1 shows the DawnRank results where the top-ranked genes with copy number aberration are listed among MMTV/Neu tumors treated with entinostat. Jun was computationally identified to be a top driver gene with copy number loss associated with resistance/progression. Not only the ranking but also the percent rank of Jun gene increased from 0.977 at 3 -week point (which means top $2.3 \%$ in the network) to 0.999 (top $0.1 \%$ ) at the time of progression. We detected 83 genes, including Jun, at Chr.4, which significantly decreased their gene expression and DNA copy number levels with q-value $0 \%$ by supervised analysis comparing the untreated samples with the samples that became resistant while on entinostat (Additional file 2: Table S4). Of note, the entirety of samples resistant to entinostat showed significantly lower copy number in a region at Chr.4 between $80,385,673$ and $101,147,931$, where Jun is located. In addition, we note a reduction of Jun gene expression levels with the duration of entinostat therapy (Fig. 4b). To test the hypothesis that Jun copy number loss causes resistance to entinostat, we sought to determine whether Jun levels influence the effects of entinostat on cell viability. Lentiviral transfection of Jun shRNA reduced Jun protein expression levels by $54-70 \%$ in BT474 and T47D (Additional file 1: Figure S3-B \& S3-C). In support of our hypothesis, reduction of Jun by shRNA imparted BT474 and T47D cells with increased resistance to entinostat (Fig. 4c, d).

\section{Genomic Jun copy number loss correlates Myc signaling activity}

Given that sensitivity to entinostat was dependent on repression of Myc target genes and that resistance to entinostat was marked by Jun loss, we examined whether loss of c-Jun restored the molecular features of Myc activation. Suggesting coordination between Myc and c-Jun, we noted that $M y c$ mRNA or signature levels increased as Jun copy number loss became deeper in MMTV/Neu samples while treated with entinostat (Fig. 3e and Fig. 4a). Therefore, we investigated the relationship between Jun copy number loss and Myc signaling in the luminal cell line BT474. Jun knockdown in BT474 correlated with upregulation of Myc gene or signature scores (Fig. 5a). To test whether our observations in preclinical models might extend to human breast cancer patients, we examined Jun copy number in human breast cancer datasets. Jun-deleted human tumors had significantly higher levels of Myc gene or signatures among both TCGA and METABRIC luminal breast cancer samples (Fig. 5b, c). All three Myc signatures were prognostic 

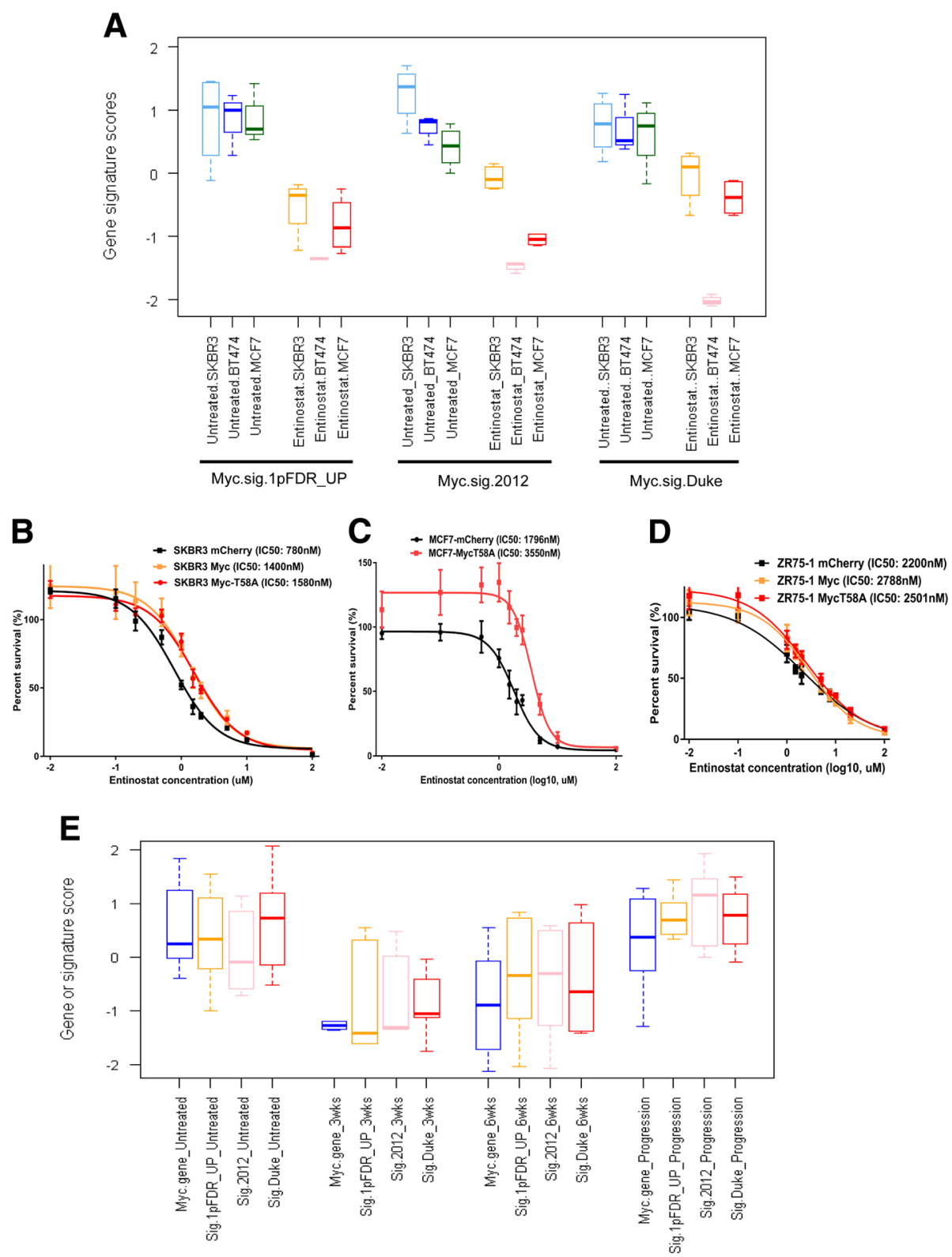

Fig. 3 Myc signaling controls entinostat sensitivity. a Myc signature analysis of entinostat-treated human breast cancer cell lines. Box and whisker plots for the Myc gene signatures (Myc.sig.1PFDR_UP, Myc.sig.2012, or Myc.sig.DUKE) using the data from SKBR3, BT474, and MCF-7 treated with or without entinostat at their $\mathrm{I}_{50}$ doses for $72 \mathrm{~h}$. Each colored square represents the relative median transcript abundance (in log2 space) of each signature for untreated SKBR3, BT474, MCF-7 being light blue, blue or light green, or entinostat-treated SKBR3, BT474, MCF-7 being green, pink, or red. b-d Sensitivity to entinostat in luminal breast cancer cell lines with lentiviral Myc constitutive overexpression. After SKBR-3 (b), MCF-7 (c), or ZR75-1 (d) cells were transfected with lentivirus-mediated Myc shRNA, the cells were incubated in various doses of entinostat for $72 \mathrm{~h}$, and then the viability of cells was measured using the MTS assay. Inhibitory concentration (IC) curves are shown with $I_{50}$ values in legend. Each point represents the mean \pm standard deviation of sextuple determinations. Myc shRNA treatment makes luminal breast cancer cells more resistant toward entinostat as evidenced by an increase in $I_{50}$. e Myc gene expression (Myc_gene) or Myc signatures (Sig.1PFDR_UP, Sig.2012, Sig.Duke) in $27 \mathrm{MMTV} / \mathrm{Neu}$ luminal mouse model tumors untreated $(N=8)$ or treated with entinostat at $12 \mathrm{mg} / \mathrm{kg}$ for 3 weeks $(N=6), 6$ weeks $(N=5)$, or until progression $(N=8)$

among luminal breast cancer samples in METABRIC as well (Fig. 5d-f), and multivariable analyses accounting for age, tumor size, nodal status, and HER2 status showed two out of three Myc signatures were still significantly prognostic (Additional file 2: Table S5). Collectively, these results suggest that genomic DNA Jun copy number loss allows activation of Myc signaling, which in turn promotes resistance to entinostat therapy. 


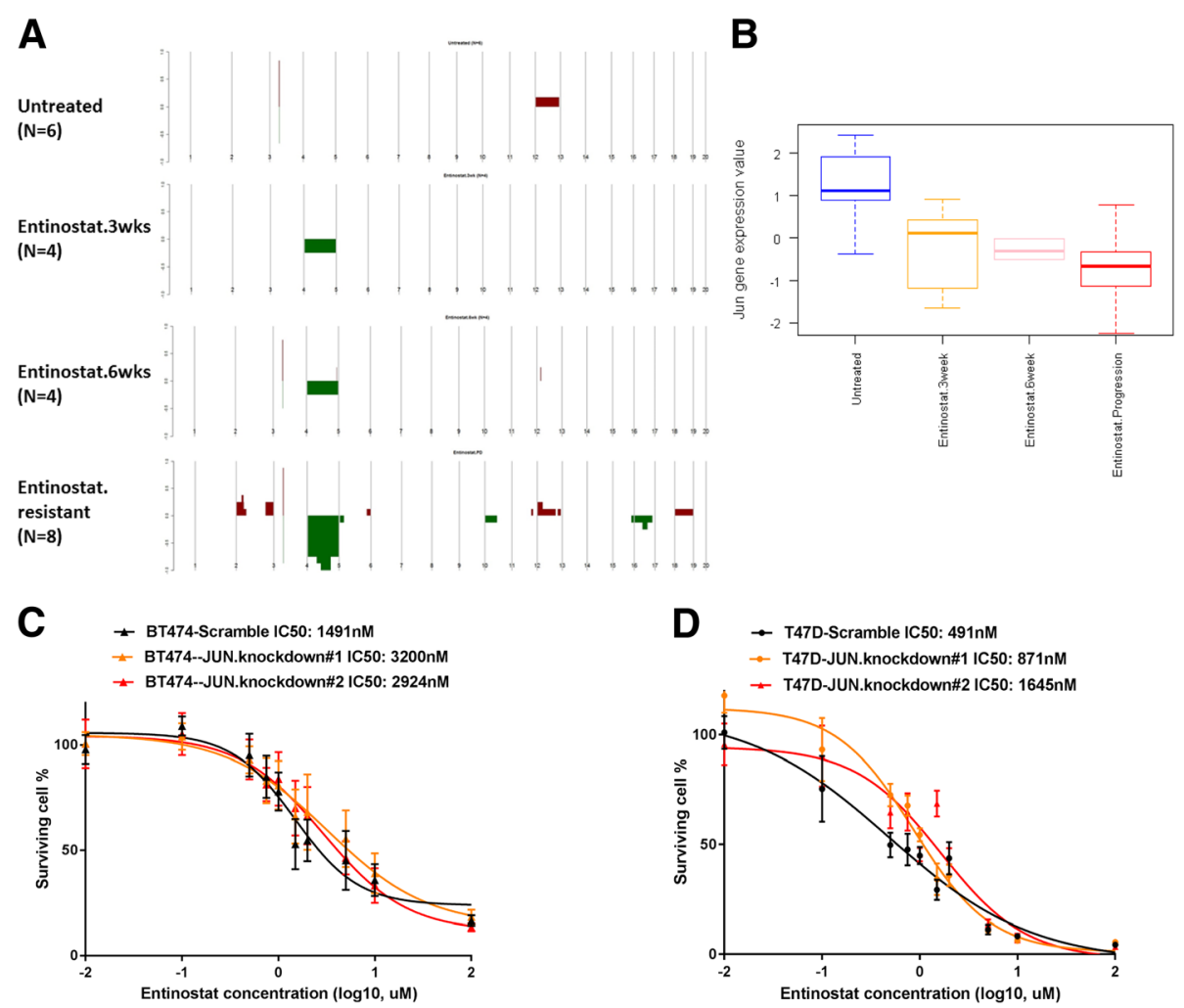

Fig. 4 Jun copy number loss causes resistance to entinostat. a Copy number landscape of entinostat-treated MMTV/Neu tumors by arrayCGH. aCGH analysis revealed that a large portion of mouse Chromosome 4 had DNA copy number loss in tumors that progressed while on entinostat. Segments of copy number gains are plotted above the $x$-axis in red and loss are plotted below the $x$-axis in green. The frequency of alterations is indicated on the $y$-axis from 0 to 100\%. b Jun gene expression in $27 \mathrm{MMTV} / \mathrm{Neu}$ luminal mouse model tumors untreated $(N=6)$ or treated with entinostat at $12 \mathrm{mg} / \mathrm{kg}$ for 3 weeks $(N=6), 6$ weeks $(N=5)$, or until progression $(N=8)$. Each square represents the relative median transcript abundance (in log2 space) of each signature for untreated or entinostat-treated MMTV/Neu. c $\mathbf{d}$ Sensitivity to entinostat in BT474 or T47D with lentiviral c-Jun knockdown. Jun shRNA treatment makes BT474 or T47D cells resistant toward entinostat as evidenced by an increase in IC ${ }_{50}$. After BT474 or T47D cells were transfected with lentivirus-mediated Jun shRNA, the cells were incubated in various doses of entinostat for $72 \mathrm{~h}$ and then the viability of cells was measured using the MTS assay. Each point represents the mean \pm standard deviation of sextuple determinations. Inhibitory concentration $(\mathrm{IC})$ curves are shown with $\mathrm{IC}_{50}$ values in legend

Table 1 DawnRank analysis of entinostat-treated MMTV/Neu samples

\begin{tabular}{|c|c|c|c|c|c|c|c|c|c|c|c|}
\hline \multicolumn{3}{|c|}{ Untreated $(N=6)$} & \multicolumn{3}{|c|}{ Entinostat-3wks $(N=4)$} & \multicolumn{3}{|c|}{ Entinostat-6wks $(N=4)$} & \multicolumn{3}{|c|}{ Entinostat-resistant $(N=8)$} \\
\hline Gene & Percent rank & Chr & Gene & Percent rank & Chr & Gene & Percent rank & Chr & Gene & Percent rank & Chr \\
\hline$\overline{M A X}$ & 1 & 12 & JUN & 0.977 & 4 & JUN & 0.997 & 4 & JUN & 0.999 & 4 \\
\hline CALM1 & 0.995 & 12 & CDC42 & 0.975 & 4 & CDC42 & 0.994 & 4 & JAK1 & 0.995 & 4 \\
\hline HIF1A & 0.990 & 12 & JAK1 & 0.969 & 4 & JAK1 & 0.992 & 4 & CDC42 & 0.990 & 4 \\
\hline ACTN1 & 0.986 & 12 & LCK & 0.969 & 4 & LCK & 0.989 & 4 & CDKN2A & 0.989 & 4 \\
\hline FOS & 0.981 & 12 & LYN & 0.965 & 4 & LYN & 0.987 & 4 & LCK & 0.987 & 4 \\
\hline HSP90AA1 & 0.976 & 12 & MAP3K7 & 0.960 & 4 & MAP3K7 & 0.984 & 4 & TGFBR1 & 0.984 & 4 \\
\hline FOXA1 & 0.972 & 12 & ERBB2 & 0.959 & 11 & CDKN2A & 0.982 & 4 & LRP8 & 0.983 & 4 \\
\hline ARF6 & 0.967 & 12 & CDKN2A & 0.952 & 4 & ZBTB17 & 0.979 & 4 & ZBTB17 & 0.981 & 4 \\
\hline FBLN5 & 0.963 & 12 & TGFBR1 & 0.950 & 4 & TGFBR1 & 0.977 & 4 & TLE1 & 0.980 & 4 \\
\hline NCOA1 & 0.958 & 12 & RBBP4 & 0.948 & 4 & RBBP4 & 0.974 & 4 & LYN & 0.980 & 4 \\
\hline NFKBIA & 0.953 & 12 & ZBTB17 & 0.944 & 4 & LRP8 & 0.972 & 4 & RBBP4 & 0.979 & 4 \\
\hline
\end{tabular}

Chr, mouse chromosome; Entinostat-3wks, Entinostat-6wks, Entinostat-resistant are samples treated with entinostat for 3 weeks, 6 weeks, and until progression, respectively 

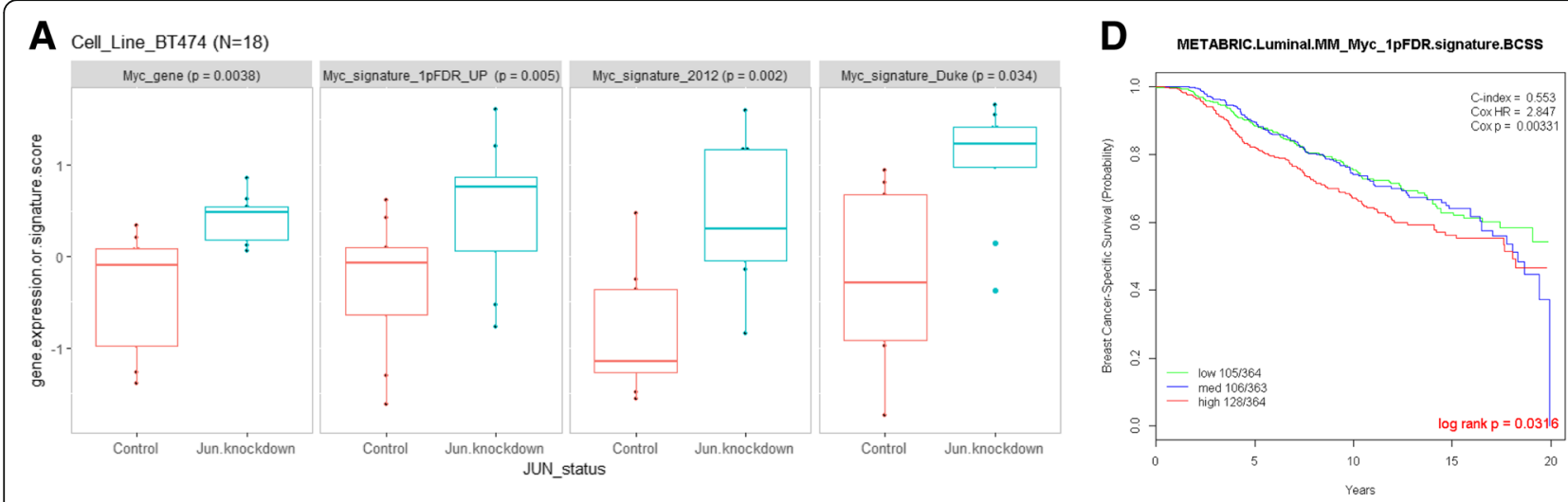

B TCGA_Luminal_A_and_Luminal_B ( $(\mathrm{N}=588)$
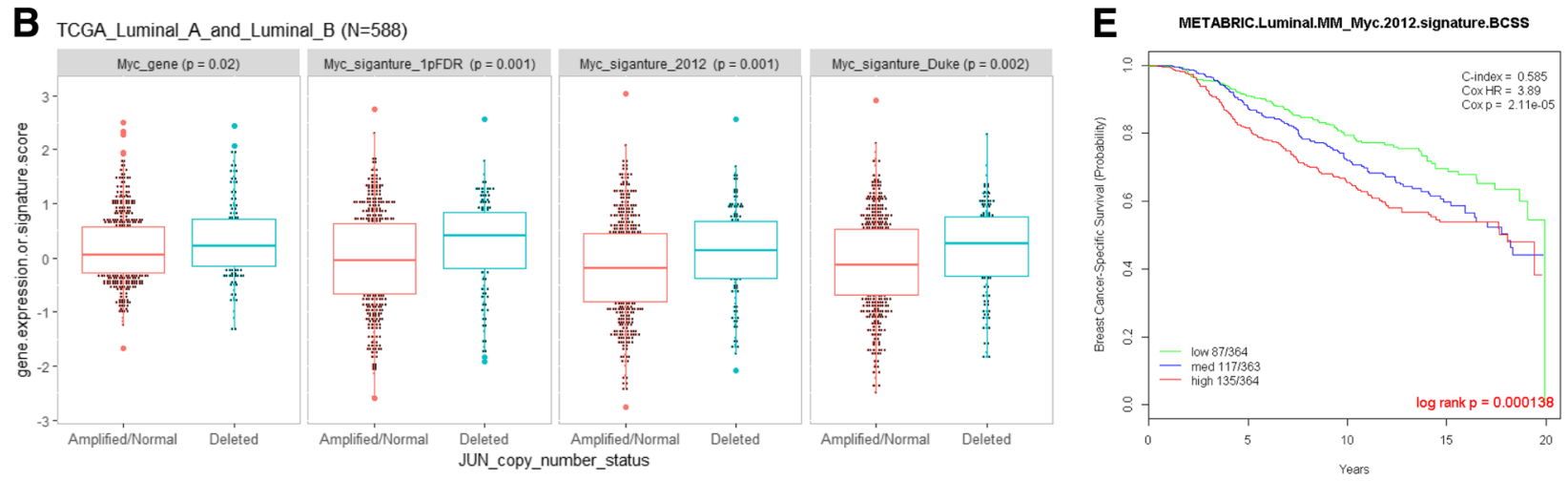

C MetABRIC_Luminal_A_and_Luminal_B $(\mathrm{N}=1206)$
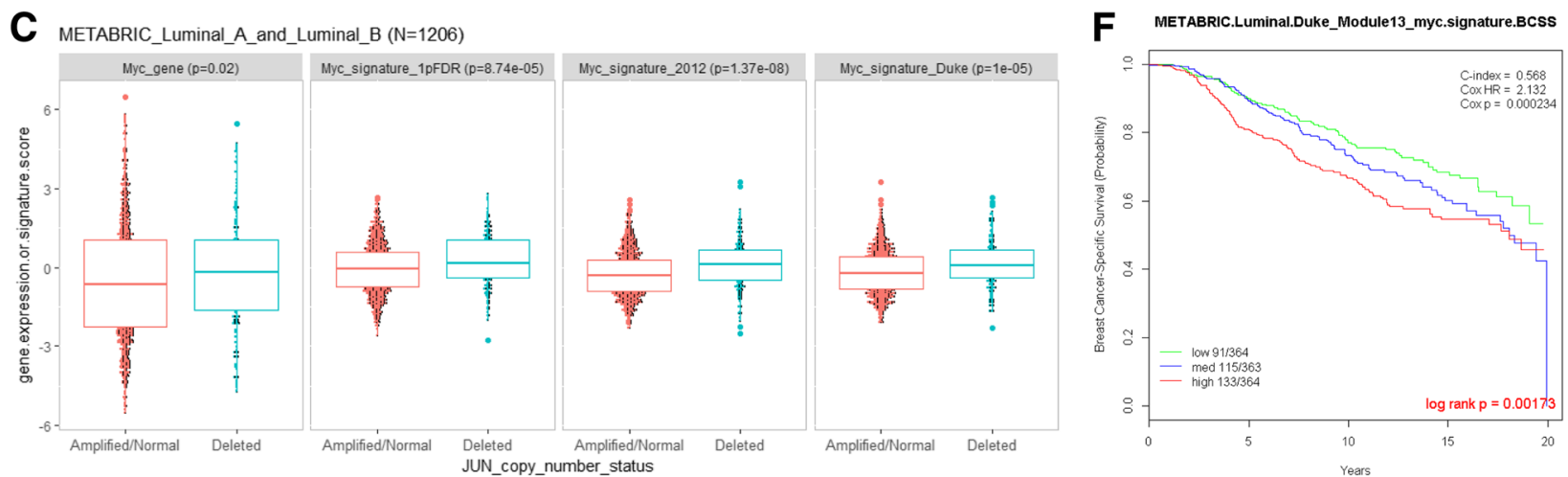

Fig. 5 Genomic Jun copy number loss correlates Myc signaling activity. a Box and whisker plots are shown as Myc gene expression (Myc.gene) or signature (1PFDR_UP, 2012, DUKE) scores in BT474 with lentiviral c-Jun or scramble shRNA (Control). The middle bar in each square represents standardized, average values. The square represents the interquartile range (25th and 75th percentiles). b Myc gene expression or signature scores in luminal TCGA breast cancer samples with Jun deletion $(N=136)$ or without Jun deletion (amplified/normal, $N=452$ ). c Myc gene expression or signature scores in luminal METABRIC breast cancer samples Jun deletion ( $N=204$ ) or without Jun deletion (amplified/normal, $N=1002$ ). d-f Kaplan-Meier plots on breast cancer-specific survival in 1091 luminal breast cancer patients in METABRIC. Patients with survival of $>20$ years were excluded. Patients were classified into three groups with lower, middle, or top third of the scores derived from the Myc signature scores using Myc_1pFDR (d), Myc.2012 (e), or Duke_Module13_myc (f). Two-sided log-rank tests and univariate Cox regression analyses were conducted to determine significance of each Myc signature

\section{Clinical impact of Jun copy number loss in human breast cancer datasets}

Jun DNA copy number values are generally lower in patients with luminal $\mathrm{A}$ and luminal $\mathrm{B}$ breast cancers (Fig. 6a). The frequencies of Jun copy number loss in luminal breast cancer were $23 \%$ and $17 \%$ in TCGA and
METABRIC, respectively (Fig. 6b). Patients with Jun copy number loss had a worse prognosis among luminal breast cancer samples in METABRIC (Fig. 6c). Further, patients with Jun copy number loss who received hormonal therapies also had worse prognosis compared with similar patients without Jun copy number loss 

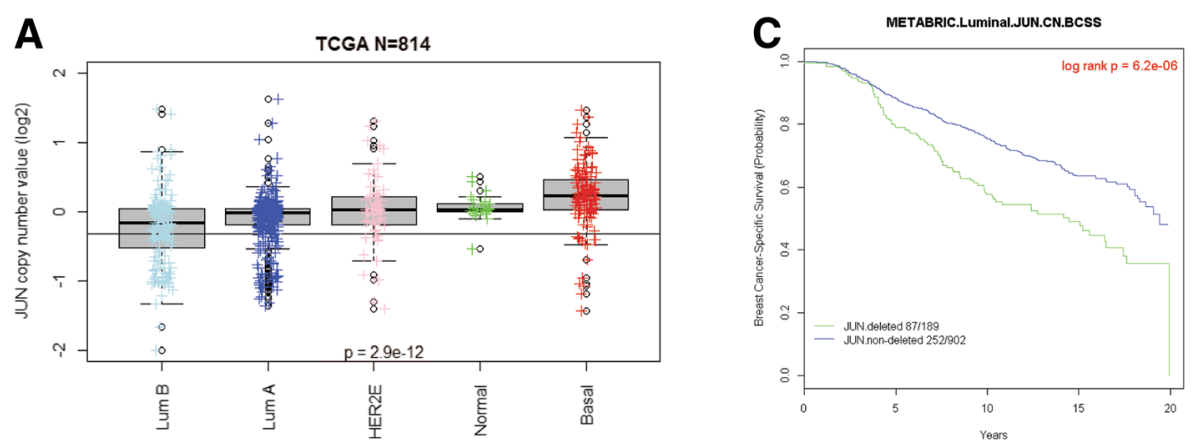

B

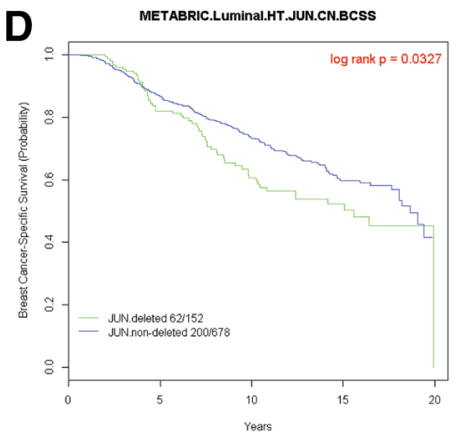

Fig. 6 Clinical impact of Jun copy number loss in human breast cancer datasets. a Log2-based value of Jun copy number across the intrinsic subtypes in 814 TCGA breast cancer patients. Whiskers represent the 5\%-95\% distribution, boxes represent the interquartile range (25th and 75 th percentiles), and the horizontal line in the box represents the median value. Cut-off value of Jun copy number for deletion was -0.32 . ANOVA $p$ value was calculated by comparing Jun copy number values across all subtypes. b Frequencies of samples with Jun deletion in TCGA ( $n=814)$ or METABRIC ( $n=1960)$ according to the intrinsic subtype. $\mathbf{c}$, $\mathbf{d}$ Kaplan-Meier plots on breast cancer-specific survival among luminal breast cancer patients in METABRIC. Patients with survival time of $>20$ years were excluded. $\mathbf{c}$ Whole luminal A and B breast cancer patients $(N=1091)$. Cox $p$ value $=8.12 \mathrm{e}-06 . \mathbf{d}$ Luminal breast cancer patients who received hormonal therapies $(N=830)$. Cox $p$ value $=0.033$

(Fig. 6d). We additionally performed survival analysis according to Jun copy number status and each Myc signature among METABRIC patients with luminal breast cancer who received hormonal therapies (Additional file 1: Figure S4). Patients with Jun-deleted/high Myc signature score had the worst prognosis (light green line), while patients with Jun-non-deleted/low Myc signature scores had the best prognosis (pink line). These results suggest that Jun copy number loss is frequently observed in human breast cancer patients, is prognostic of worse outcomes, and might be a genetic cause of resistance to entinostat among patients with luminal tumors who receive hormonal therapies.

\section{Discussion}

With our work, we identify a complex interplay between the HDAC inhibitor entinostat, c-Jun, and Myc. Preclinical models, both human and murine, of luminal subtypes exhibited sensitivity to entinostat compared to basal-like or claudin-low subtypes. Importantly, we show that entinostat inhibited luminal breast cancer through Myc signaling, and genomic Jun loss upregulated Myc signaling to promote resistance to entinostat. Our findings indicate that Jun copy number loss might, therefore, represent a useful biomarker for entinostat resistance in luminal breast cancer where these Jun-deleted patients might be suggested to not receive entinostat containing regimens. Another alternative biomarker for entinostat resistance could also be high Myc signature expression. These novel findings are to the best of our knowledge, have not been previously reported.

$M y c$ is the most frequently amplified oncogene [48] and it is known to regulate transcription of genes involved in cell growth and proliferation [49, 50]. The functions of Myc is influenced by multiple mechanisms in tumor cells: protein ubiquitination, gene amplification, chromosomal translocation, mutation, co-factor expression, and mutation of upstream signaling pathways [51-55]. Therefore, transcriptional or genomic levels of $M y c$ itself do not always reflect on its activity. Gene signatures of Myc target genes might better reflect on Myc activity; however, Myc amplifies thousands of actively transcribed genes within each cell type, so every cancer has a different, specific cohort of Myc target genes [56, 57]. This is the reason why we investigated a number of different $M y c$ signatures, all of whom were derived from breast epithelial tissues, and all were prognostic in patients with luminal breast cancer (Fig. 6). In our study, Myc signatures were repressed while breast epithelial cells were responsive on entinostat, and Myc signatures were reactivated upon 
progression in vivo. Myc signatures were repressed after entinostat treatment at $\mathrm{IC}_{50}$ doses and Myc overexpression resulted in resistance to entinostat in vitro. These data support the hypothesis that Myc represents an important modulator for response to entinostat as extensive evidence on other HDAC inhibitors has suggested [12-15].

This study also revealed that a specific region of mouse chromosome 4 was recurrently deleted in every entinostat-resistant MMTV/Neu tumor obtained $(n=8)$, but not in untreated tumors. This deletion has been reported in a variety of luminal breast cancer mouse models like MMTV/Neu and p53 null luminal tumors $[25,39,58,59]$. Major human counterparts of mouse chromosome 4 are Chr.1p31-36 and Chr.9; loss of heterozygosity on $1 \mathrm{p}$ (where c-Jun is) occurred preferentially in a subclass of estrogen receptor-positive breast cancers [60] and has been shown as a poor prognostic factor [61-63]. These findings imply that deleted mouse chromosome 4 drives tumor aggressiveness, and this is a region also linked to poor outcomes in human luminal breast cancers as well.

To identify possible drivers in this luminal tumor conserved region of deletion, we used a network-based, integrated bioinformatics analysis (i.e., DawnRank) that identified Jun deletion at mouse chromosome 4 as the top driver gene upon progression during entinostat treatment. Knockdown of Jun expression in luminal cells increased resistance to entinostat (Fig. 4c, d), and genomic loss of Jun at 1p32, which was found in $17-23 \%$ of patients with luminal breast cancer, was significantly prognostic among patients with luminal breast cancer who received anti-hormonal therapies (Fig. 6). Furthermore, we found Jun-deleted samples had higher Myc signature scores in vitro and in vivo in human breast cancer (Fig. 5). Similar findings have been found between loss of heterozygosity of chromosome 1p32-pter and amplification of $M y c$ [64]. The precise molecular mechanism(s) as to how Jun deletion causes upregulation of Myc signaling remains unclear, but the interplay between Jun and Myc has been previously reported $[65,66]$. In detail, Jun/Ap-1 complex might regulate Myc directly [65] or Jun loss may modulate Myc function indirectly [66]. We would propose a mechanism that genomic Jun loss constitutively activates Myc signaling, which leads to poor outcomes in general, and possible resistance to entinostat. Measuring Jun DNA copy number loss is one of the candidate biomarkers for a clinical test, if entinostat achieves approval. Alternatively, gene expression of Myc signatures are also a clinical test candidate; those who have high Myc signature would be predicted to have resistance to entinostat. Lastly, we will need retrospective analysis of existing clinical trials to determine which one is the best.

\section{Conclusions}

Entinostat inhibited luminal breast cancer through Myc signaling, which was upregulated by Jun DNA loss to promote resistance to entinostat in our models. Here we also provide a testing platform using MMTV/Neu with genomic Jun loss for combination therapies with entinostat to provide more durable response to Jun-deleted human luminal breast cancer. Further studies will be certainly required to validate the significance of genomic Jun loss in prospectively collected luminal breast cancer samples under treatment of entinostat.

\section{Additional files}

Additional file 1: Figure S1. Long-term survival results for eight mouse models of basal breast cancer. Figure S2. Analysis of potential entinostat gene signatures. Figure S3. Western blotting results on lentiviral transfection of Myc or shJun. Figure S4. Clinical impact of Jun copy number loss in METABRIC. (DOCX $8120 \mathrm{~kb})$

Additional file 2: Table S1. Genes in mouse and human version of Myc signatures. Table S2. A. Upregulated and downregulated genes with FDR of $0 \%$ in SAM analysis. B: Entinostat signatures. Table S3. The changes in tumor size of MMTV/Neu tumors treated with entinostat. Table S4. 83 genes which significantly decreased at gene expression and copy number levels with q-value $0 \%$ by SAM analysis comparing the untreated samples and the samples resistant while on entinostat. Table S5. Multivariate analysis of each Myc signature accounting for clinical features in METABRIC. (XLSX 74 kb)

\section{Acknowledgements}

We thank Gary Johnson, Matthew Ellis, and Jon Serody for gene constructs or cell lines used in this study.

\section{Funding}

This work was supported by funds from the $\mathrm{NCI}$ Breast SPORE program (P50-CA58223), RO1-CA148761, RO1-CA195740, by the Breast Cancer Research Foundation, and by the Susan G. Komen to Charles M. Perou (SAC-160074) and Maki Tanioka (PDF16378265).

\section{Availability of data and materials}

All genomic data, including DNA copy number and gene expression, have been deposited into the GEO (series ID GSE118744). The results published here are in whole or part based upon data from the Cancer Genome Atlas managed by the $\mathrm{NCl}$ and NHGRI (dbGaP accession phs000178).

\section{Authors' contributions}

MT analyzed and interpreted the in vitro, in vivo, and patient data regarding the entinostat experiments and CF assisted these analyses. KRM, DPH, and DBD performed the experiments on genetically engineered mice using entinostat, and MT and CMP were major contributors in writing the manuscript. All authors read and approved the final manuscript.

\section{Ethics approval}

All animal work was done under protocols approved by the University of North Carolina (UNC; Chapel Hill, NC) Institutional Animal Care and Use Committee.

\section{Consent for publication}

Not applicable.

\section{Competing interests}

The following authors indicated a financial interest. Research support: none. Ownership: Charles M Perou, Bioclassifier, GeneCentric Diagnostics. Income: Charles M Perou, royalties from PAM50 breast cancer gene patent application and from lung gene signature patent. Intellectual Property: Charles M Perou, PAM50 breast cancer gene patent. The remaining authors declare that they have no competing interests. 


\section{Publisher's Note}

Springer Nature remains neutral with regard to jurisdictional claims in published maps and institutional affiliations.

\section{Author details \\ ${ }^{1}$ Lineberger Comprehensive Cancer Center, University of North Carolina at Chapel Hill, Chapel Hill, NC 27599, USA. ${ }^{2}$ Department of Genetics, University of North Carolina, Chapel Hill, NC 27599, USA. ${ }^{3}$ Lineberger Comprehensive Hill, Chapel Hill, NC 27599, USA. \\ Received: 5 September 2018 Accepted: 8 November 2018 Published online: 30 November 2018} Cancer Center, The Animal Study Core, University of North Carolina at Chapel

\section{References}

1. Torre LA, Bray F, Siegel RL, Ferlay J, Lortet-Tieulent J, Jemal A. Global cancer statistics, 2012. CA Cancer J Clin. 2015;65:87-108.

2. Finn RS, Crown JP, Lang I, Boer K, Bondarenko IM, Kulyk SO, Ettl J, Patel R, Pinter T, Schmidt M, et al. The cyclin-dependent kinase 4/6 inhibitor palbociclib in combination with letrozole versus letrozole alone as first-line treatment of oestrogen receptor-positive, HER2-negative, advanced breast cancer (PALOMA-1/TRIO-18): a randomised phase 2 study. Lancet Oncol. 2015;16:25-35

3. Hortobagyi GN, Stemmer SM, Burris HA, Yap YS, Sonke GS, Paluch-Shimon S, Campone M, Blackwell KL, Andre F, Winer EP, et al. Ribociclib as first-line therapy for HR-positive, advanced breast Cancer. N Engl J Med. 2016;375: 1738-48.

4. Baselga J, Campone M, Piccart M, Burris HA 3rd, Rugo HS, Sahmoud T, Noguchi S, Gnant M, Pritchard Kl, Lebrun F, et al. Everolimus in postmenopausal hormone-receptor-positive advanced breast cancer. N Engl J Med. 2012;366:520-9.

5. Ryan RJ, Bernstein BE. Molecular biology. Genetic events that shape the cancer epigenome. Science. 2012;336:1513-4.

6. Muller BM, Jana L, Kasajima A, Lehmann A, Prinzler J, Budczies J, Winzer KJ, Dietel $\mathrm{M}$, Weichert W, Denkert C. Differential expression of histone deacetylases HDAC1, 2 and 3 in human breast cancer--overexpression of HDAC2 and HDAC3 is associated with clinicopathological indicators of disease progression. BMC Cancer. 2013;13:215.

7. Sabnis GJ, Goloubeva OG, Kazi AA, Shah P, Brodie AH. HDAC inhibitor entinostat restores responsiveness of letrozole-resistant MCF-7Ca xenografts to aromatase inhibitors through modulation of Her-2. Mol Cancer Ther. 2013;12:2804-16.

8. Benedetti R, Conte M, Altucci L. Targeting histone deacetylases in diseases: where are we? Antioxid Redox Signal. 2015;23:99-126.

9. San-Miguel JF, Hungria VT, Yoon SS, Beksac M, Dimopoulos MA, Elghandour A, Jedrzejczak WW, Gunther A, Nakorn TN, Siritanaratkul N, et al. Panobinostat plus bortezomib and dexamethasone versus placebo plus bortezomib and dexamethasone in patients with relapsed or relapsed and refractory multiple myeloma: a multicentre, randomised, double-blind phase 3 trial. Lancet Oncol. 2014;15:1195-206.

10. Piekarz RL, Frye R, Turner M, Wright JJ, Allen SL, Kirschbaum MH, Zain J, Prince HM, Leonard JP, Geskin LJ, et al. Phase II multi-institutional trial of the histone deacetylase inhibitor romidepsin as monotherapy for patients with cutaneous T-cell lymphoma. J Clin Oncol. 2009;27:5410-7.

11. Yardley DA, Ismail-Khan RR, Melichar B, Lichinitser M, Munster PN, Klein PM, Cruickshank S, Miller KD, Lee MJ, Trepel JB. Randomized phase II, doubleblind, placebo-controlled study of exemestane with or without entinostat in postmenopausal women with locally recurrent or metastatic estrogen receptor-positive breast cancer progressing on treatment with a nonsteroidal aromatase inhibitor. J Clin Oncol. 2013;31:2128-35.

12. Marshall GM, Gherardi S, Xu N, Neiron Z, Trahair T, Scarlett CJ, Chang DK, Liu PY, Jankowski K, Iraci N, et al. Transcriptional upregulation of histone deacetylase 2 promotes Myc-induced oncogenic effects. Oncogene. 2010; 29:5957-68.

13. Labisso WL, Wirth M, Stojanovic N, Stauber RH, Schnieke A, Schmid RM, Kramer OH, Saur D, Schneider G. MYC directs transcription of MCL1 and elF4E genes to control sensitivity of gastric cancer cells toward HDAC inhibitors. Cell Cycle. 2012;11:1593-602.

14. Nebbioso A, Carafa V, Conte M, Tambaro FP, Abbondanza C, Martens J, Nees M, Benedetti R, Pallavicini I, Minucci S, et al. c-Myc modulation and acetylation is a key HDAC inhibitor target in cancer. Clin Cancer Res. 2017; 23(10):2542-55.

15. Pei Y, Liu KW, Wang J, Garancher A, Tao R, Esparza LA, Maier DL, Udaka YT, Murad N, Morrissy S, et al. HDAC and PI3K antagonists cooperate to inhibit growth of MYC-driven Medulloblastoma. Cancer Cell. 2016;29:311-23.

16. Lee J, Bartholomeusz C, Mansour O, Humphries J, Hortobagyi GN, Ordentlich P, Ueno NT. A class I histone deacetylase inhibitor, entinostat, enhances lapatinib efficacy in HER2-overexpressing breast cancer cells through FOXO3-mediated Bim1 expression. Breast Cancer Res Treat. 2014; 146:259-72.

17. Shah P, Gau Y, Sabnis G. Histone deacetylase inhibitor entinostat reverses epithelial to mesenchymal transition of breast cancer cells by reversing the repression of E-cadherin. Breast Cancer Res Treat. 2014;143:99-111.

18. Kim K, Skora AD, Li Z, Liu Q, Tam AJ, Blosser RL, Diaz LA Jr, Papadopoulos N, Kinzler KW, Vogelstein B, Zhou S. Eradication of metastatic mouse cancers resistant to immune checkpoint blockade by suppression of myeloidderived cells. Proc Natl Acad Sci U S A. 2014;111:11774-9.

19. Orillion A, Hashimoto A, Damayanti NP, Shen L, Adelaiye-Ogala R, Arisa S, Chintala S, Ordentlich P, Kao C, Elzey B, et al. Entinostat neutralizes myeloid derived suppressor cells and enhances the antitumor effect of PD-1 inhibition in murine models of lung and renal cell carcinoma. Clin Cancer Res. 2017.

20. Troester MA, Hoadley KA, Sorlie T, Herbert BS, Borresen-Dale AL, Lonning PE, Shay JW, Kaufmann WK, Perou CM. Cell-type-specific responses to chemotherapeutics in breast cancer. Cancer Res. 2004;64:4218-26.

21. Herschkowitz JI, Simin K, Weigman VJ, Mikaelian I, Usary J, Hu Z, Rasmussen KE, Jones LP, Assefnia S, Chandrasekharan S, et al. Identification of conserved gene expression features between murine mammary carcinoma models and human breast tumors. Genome Biol. 2007;8:R76.

22. Katherine A Hoadley WTB, Brandelyn N Pitcher, Joel S Parker, Matthew D Wilkerson, William Irvin, Jr1, Norah, Lynn Henry SMT, Chau Dang, lan E Krop, Donald A Berry, Elaine R Mardis, Charles M Perou, Eric P Winer, Clifford A Hudis LAC: Mutational analysis of CALGB 40601 (Alliance), a neoadjuvant phase III trial of weekly paclitaxel $(T)$ and trastuzumab $(H)$ with or without lapatinib (L) for HER2-positive breast cancer. In San Antonio Breast Cancer Symposium San Antonio 2014: S3-08.

23. Maroulakou IG, Anver M, Garrett L, Green JE. Prostate and mammary adenocarcinoma in transgenic mice carrying a rat C3(1) simian virus 40 large tumor antigen fusion gene. Proc Natl Acad Sci U S A. 1994;91:11236-40.

24. Li S, Shen D, Shao J, Crowder R, Liu W, Prat A, He X, Liu S, Hoog J, Lu C, et al. Endocrine-therapy-resistant ESR1 variants revealed by genomic characterization of breast-cancer-derived xenografts. Cell Rep. 2013;4:1116-30.

25. Herschkowitz JI, Zhao W, Zhang M, Usary J, Murrow G, Edwards D, Knezevic J, Greene SB, Darr D, Troester MA, et al. Comparative oncogenomics identifies breast tumors enriched in functional tumor-initiating cells. Proc Natl Acad Sci U S A. 2012;109:2778-83.

26. Jerry DJ, Kittrell FS, Kuperwasser C, Laucirica R, Dickinson ES, Bonilla PJ, Butel JS, Medina D. A mammary-specific model demonstrates the role of the p53 tumor suppressor gene in tumor development. Oncogene. 2000;19:1052-8

27. Hu Z, Fan C, Oh DS, Marron JS, He X, Qaqish BF, Livasy C, Carey LA, Reynolds E, Dressler $L$, et al. The molecular portraits of breast tumors are conserved across microarray platforms. BMC Genomics. 2006;7:96.

28. Tusher VG, Tibshirani R, Chu G. Significance analysis of microarrays applied to the ionizing radiation response. Proc Natl Acad Sci U S A. 2001:98:5116-21.

29. Ciriello G, Gatza ML, Beck AH, Wilkerson MD, Rhie SK, Pastore A, Zhang H, McLellan M, Yau C, Kandoth C, et al. Comprehensive molecular portraits of invasive lobular breast Cancer. Cell. 2015;163:506-19.

30. Perou CM, Sorlie T, Eisen MB, van de Rijn M, Jeffrey SS, Rees CA, Pollack JR, Ross DT, Johnsen H, Akslen LA, et al. Molecular portraits of human breast tumours. Nature. 2000;406:747-52.

31. Saldanha AJ. Java Treeview--extensible visualization of microarray data. Bioinformatics. 2004;20:3246-8.

32. Subramanian A, Tamayo P, Mootha VK, Mukherjee S, Ebert BL, Gillette MA, Paulovich A, Pomeroy SL, Golub TR, Lander ES, Mesirov JP. Gene set enrichment analysis: a knowledge-based approach for interpreting genome-wide expression profiles. Proc Natl Acad Sci U S A. 2005;102: $15545-50$.

33. Tanioka MFC, Parker JS, Hoadley KA, Hu Z, Li Y, Hyslop T, Pitcher BN, Soloway M, Spears PA, et al. Integrated analysis of RNA and DNA from the phase III trial CALGB 40601 identifies predictors of response to trastuzumab- 
based neoadjuvant chemotherapy in HER2-positive breast cancer. Clinical Cancer Research. 2018; in press.

34. Tanioka M, Fan C, Parker JS, Hoadley KA, Hu Z, Li Y, Hyslop TM, Pitcher BN, Soloway MG, Spears PA, et al. Integrated analysis of RNA and DNA from the phase III trial CALGB 40601 identifies predictors of response to Trastuzumabbased neoadjuvant chemotherapy in HER2-positive breast Cancer. Clin Cancer Res. 2018.

35. Fan C, Prat A, Parker JS, Liu Y, Carey LA, Troester MA, Perou CM. Building prognostic models for breast cancer patients using clinical variables and hundreds of gene expression signatures. BMC Med Genet. 2011;4:3.

36. Pfefferle AD, Herschkowitz JI, Usary J, Harrell JC, Spike BT, Adams JR, TorresArzayus MI, Brown M, Egan SE, Wahl GM, et al. Transcriptomic classification of genetically engineered mouse models of breast cancer identifies human subtype counterparts. Genome Biol. 2013;14:R125.

37. Gatza ML, Lucas JE, Barry WT, Kim JW, Wang Q, Crawford MD, Datto MB, Kelley M, Mathey-Prevot B, Potti A, Nevins JR. A pathway-based classification of human breast cancer. Proc Natl Acad Sci U S A. 2010;107:6994-9.

38. Weigman VJ, Chao HH, Shabalin AA, He X, Parker JS, Nordgard SH, Grushko T, Huo D, Nwachukwu C, Nobel A, et al. Basal-like breast cancer DNA copy number losses identify genes involved in genomic instability, response to therapy, and patient survival. Breast Cancer Res Treat. 2012;133:865-80.

39. Silva GO, He X, Parker JS, Gatza ML, Carey LA, Hou JP, Moulder SL, Marcom PK, Ma J, Rosen JM, Perou CM. Cross-species DNA copy number analyses identifies multiple 1q21-q23 subtype-specific driver genes for breast cancer. Breast Cancer Res Treat. 2015;152:347-56.

40. Hou JP, Ma J. DawnRank: discovering personalized driver genes in cancer. Genome Med. 2014;6:56.

41. Curtis C, Shah SP, Chin SF, Turashvili G, Rueda OM, Dunning MJ, Speed D, Lynch AG, Samarajiwa S, Yuan Y, et al. The genomic and transcriptomic architecture of 2,000 breast tumours reveals novel subgroups. Nature. 2012; 486:346-52.

42. Silva GO, Siegel MB, Mose LE, Parker JS, Sun W, Perou CM, Chen M. SynthEx: a synthetic-normal-based DNA sequencing tool for copy number alteration detection and tumor heterogeneity profiling. Genome Biol. 2017;18:66.

43. Quinlan AR, Hall IM. BEDTools: a flexible suite of utilities for comparing genomic features. Bioinformatics. 2010;26:841-2.

44. Cancer Genome Atlas N. Comprehensive molecular portraits of human breast tumours. Nature. 2012;490:61-70.

45. Beroukhim R, Getz G, Nghiemphu L, Barretina J, Hsueh T, Linhart D, Vivanco I, Lee JC, Huang JH, Alexander $\mathrm{S}$, et al. Assessing the significance of chromosomal aberrations in cancer: methodology and application to glioma. Proc Natl Acad Sci U S A. 2007;104:20007-12.

46. Sorlie T, Perou CM, Tibshirani R, Aas T, Geisler S, Johnsen H, Hastie T, Eisen $M B$, van de Rijn M, Jeffrey SS, et al. Gene expression patterns of breast carcinomas distinguish tumor subclasses with clinical implications. Proc Natl Acad Sci U S A. 2001;98:10869-74.

47. Zhu P, Martin E, Mengwasser J, Schlag P, Janssen KP, Gottlicher M. Induction of HDAC2 expression upon loss of APC in colorectal tumorigenesis. Cancer Cell. 2004:5:455-63.

48. Beroukhim R, Mermel CH, Porter D, Wei G, Raychaudhuri S, Donovan J, Barretina J, Boehm JS, Dobson J, Urashima M, et al. The landscape of somatic copy-number alteration across human cancers. Nature. 2010;463: 899-905.

49. Dang CV. MYC on the path to cancer. Cell. 2012;149:22-35.

50. Adhikary S, Eilers M. Transcriptional regulation and transformation by Myc proteins. Nat Rev Mol Cell Biol. 2005;6:635-45.

51. Eilers M, Eisenman RN. Myc's broad reach. Genes Dev. 2008;22:2755-66.

52. Meyer N, Penn LZ. Reflecting on 25 years with MYC. Nat Rev Cancer. 2008;8: 976-90

53. Pomerantz MM, Ahmadiyeh N, Jia L, Herman P, Verzi MP, Doddapaneni $H$, Beckwith CA, Chan JA, Hills A, Davis M, et al. The 8q24 cancer risk variant rs6983267 shows long-range interaction with MYC in colorectal cancer. Nat Genet. 2009:41:882-4.

54. Wright JB, Brown SJ, Cole MD. Upregulation of C-MYC in cis through a large chromatin loop linked to a cancer risk-associated single-nucleotide polymorphism in colorectal cancer cells. Mol Cell Biol. 2010;30:1411-20.

55. Reavie L, Buckley SM, Loizou E, Takeishi S, Aranda-Orgilles B, Ndiaye-Lobry D, Abdel-Wahab O, Ibrahim S, Nakayama Kl, Aifantis I. Regulation of c-Myc ubiquitination controls chronic myelogenous leukemia initiation and progression. Cancer Cell. 2013;23:362-75.
56. Nie Z, Hu G, Wei G, Cui K, Yamane A, Resch W, Wang R, Green DR, Tessarollo L, Casellas R, et al. c-Myc is a universal amplifier of expressed genes in lymphocytes and embryonic stem cells. Cell. 2012;151:68-79.

57. Lin CY, Loven J, Rahl PB, Paranal RM, Burge CB, Bradner JE, Lee TI, Young RA. Transcriptional amplification in tumor cells with elevated c-Myc. Cell. 2012;151:56-67.

58. Montagna C, Andrechek ER, Padilla-Nash H, Muller WJ, Ried T. Centrosome abnormalities, recurring deletions of chromosome 4 , and genomic amplification of HER2/neu define mouse mammary gland adenocarcinomas induced by mutant HER2/neu. Oncogene. 2002;21:890-8.

59. Hodgson JG, Malek T, Bornstein S, Hariono S, Ginzinger DG, Muller WJ, Gray JW. Copy number aberrations in mouse breast tumors reveal loci and genes important in tumorigenic receptor tyrosine kinase signaling. Cancer Res. 2005;65:9695-704

60. Wang ZC, Lin M, Wei L, Li C, Miron A, Lodeiro G, Harris L, Ramaswamy S, Tanenbaum DM, Meyerson M, et al. Loss of heterozygosity and its correlation with expression profiles in subclasses of invasive breast cancers. Cancer Res. 2004;64:64-71.

61. Ragnarsson G, Sigurdsson A, Eiriksdottir G, Barkardottir R, Jonasson J, Ingvarsson S. Loss of heterozygosity at chromosome $1 p$ in human breast cancer. Int J Oncol. 1996;9:731-6.

62. Ragnarsson G, Eiriksdottir G, Johannsdottir JT, Jonasson JG, Egilsson V, Ingvarsson S. Loss of heterozygosity at chromosome $1 \mathrm{p}$ in different solid human tumours: association with survival. Br J Cancer. 1999;79:1468-74.

63. Borg A, Zhang QX, Olsson H, Wenngren E. Chromosome 1 alterations in breast cancer: allelic loss on $1 \mathrm{p}$ and $1 \mathrm{q}$ is related to lymphogenic metastases and poor prognosis. Genes Chromosomes Cancer. 1992;5:311-20.

64. Bieche I, Champeme MH, Lidereau R. A tumor suppressor gene on chromosome 1p32-pter controls the amplification of MYC family genes in breast cancer. Cancer Res. 1994:54:4274-6.

65. Vartanian R, Masri J, Martin J, Cloninger C, Holmes B, Artinian N, Funk A, Ruegg T, Gera J. AP-1 regulates cyclin D1 and c-MYC transcription in an AKT-dependent manner in response to MTOR inhibition: role of AIP4/itchmediated JUNB degradation. Mol Cancer Res. 2011;9:115-30.

66. Vaque JP, Fernandez-Garcia B, Garcia-Sanz P, Ferrandiz N, Bretones G, Calvo F, Crespo P, Marin MC, Leon J. c-Myc inhibits Ras-mediated differentiation of pheochromocytoma cells by blocking c-Jun up-regulation. Mol Cancer Res. 2008;6:325-39.

Ready to submit your research? Choose BMC and benefit from:

- fast, convenient online submission

- thorough peer review by experienced researchers in your field

- rapid publication on acceptance

- support for research data, including large and complex data types

- gold Open Access which fosters wider collaboration and increased citations

- maximum visibility for your research: over $100 \mathrm{M}$ website views per year

At BMC, research is always in progress.

Learn more biomedcentral.com/submissions 